\title{
Small Functions of Meromorphic Functions that Share Three Values GCM
}

\author{
Thamir ChilaAp Alzahary \\ Department of Mathematics, Shanghai University, Shanghai 200444, China \\ e-mail : thammra@yahoo.com
}

ABstract. In this paper, we deal with the problem of uniqueness of meromorphic functions that share three values, and obtain some theorems which improve some results of Brosch, Yi and other authors.

\section{Introduction and definitions}

Let $f$ and $g$ be two nonconstant meromorphic functions on the open complex plane $\mathbb{C}$, and let $a$ be a finite value in the complex plane. We say that $f$ and $g$ share the value $a C M(I M)$ provided that $f-a$ and $g-a$ have the same zeros counting multiplicities ( ignoring multiplicities ), and $f, g$ share $\infty C M(I M)$ provided that $1 / f, 1 / g$ share 0 CM ( IM ). We do not explain the standard notations of value distribution theory as those are available in Hayman [4] or Yang and Yi [11].

We denote by $S(r, f)$ any function satisfying $S(r, f)=o(T(r, f))$ as $r \rightarrow+\infty$ possibly outside a set $E$ of finite Lebesgue measure. A meromorphic function $a(z)$ is said to be a small function of $f$, if $T(r, a)=S(r, f)$.

Let $f$ and $g$ be nonconstant meromorphic functions and $a$ be a small meromorphic function of $f$ and $g$. We denote by $\bar{N}(r, a, f, g)\left(\right.$ and $\left.\bar{N}_{E}(r, a, f, g)\right)$ the reduce counting function of the common zeros of $f-a$ and $g-a$ (with the same multiplicities). We write $f=a \Rightarrow g=a$ to mean that $\bar{N}\left(r, \frac{1}{f-a}\right)-\bar{N}(r, a, f, g)=S(r, f)$. We say that $f$ and $g$ share a GIM (some authors use the symbol $I M^{*}$ or "IM"), if $f=a \Rightarrow g=a$ and $g=a \Rightarrow f=a$. If

$\bar{N}\left(r, \frac{1}{f-a}\right)-\bar{N}_{E}(r, a, f, g)=S(r, f)$ and $\bar{N}\left(r, \frac{1}{g-a}\right)-\bar{N}_{E}(r, a, f, g)=S(r, g)$,

then we say that $f$ and $g$ share a GCM (some authors use the symbol $C M^{*}$ or "CM" )(see ([8], [11], [15])). Evidently, if $f$ and $g$ share $a$ IM (or CM) then $f$ and $g$ share a GIM ( or GCM ).

Received 15 October 2007; revised 22 April 2008; accepted 21 April 2008.

2000 Mathematics Subject Classification: 30D35, 30D30.

Key words and phrases: meromorphic functions, weighted sharing, meromorphic functions, small functions.

The research was partially supported by Shanghai Leading Academic Discipline Project, China (J50101). 
Definition 1. Let $p$ be a positive integer. We denote by $N_{p)}(r, f)\left(\right.$ or $\left.\bar{N}_{p)}(r, f)\right)$ the counting function of all poles of $f$ with multiplicities $\leq p$ ( ignoring multiplicities). We recall that $N_{(p+1}(r, f)=N(r, f)-N_{p)}(r, f)$ and $\bar{N}_{(p+1}(r, f)=\bar{N}(r, f)-$ $\bar{N}_{p)}(r, f)$.

Lahiri [5] introduced the notion of weighted sharing by the following definition:

Definition 2. Let $k$ be a nonnegative integer or infinity. For any $a \in C \bigcup\{\infty\}$, we denote by $E_{k}(a, f)$ the set of all $a$-points of $f$, where an $a$-point of multiplicity $m$ is counted $m$ times if $m \leq k$ and $k+1$ times if $m>k$. If $E_{k}(a, f)=E_{k}(a, g)$, we say that $f, g$ share $(a, k)$.

Yi [13] proved the following theorem which is extended the results of Ueda [10] and $\mathrm{Ye}[12]$.

Theorem A. Let $f$ and $g$ be two distinct nonconstant meromorphic functions sharing $0,1, \infty C M$, and let $a(\neq 0,1)$ be a finite complex number. If $N\left(r, \frac{1}{g-a}\right) \neq$ $T(r, g)+S(r, g)$, then a is a Picard exceptional value of $g$, and $f$ and $g$ satisfy one of the following three relations:

(i) $(g-a)(f+a-1) \equiv a(1-a)$; (ii) $g+(a-1) f \equiv a$; (iii) $g \equiv a f$.

Recently, the author [1] has proved the following two results.

Theorem B. Let $f$ and $g$ be two distinct nonconstant meromorphic functions sharing $\left(0, k_{1}\right),\left(1, k_{2}\right),\left(\infty, k_{3}\right)$, where $k_{j}(j=1,2,3)$ are positive integers satisfying

$$
k_{1} k_{2} k_{3}>k_{1}+k_{2}+k_{3}+2 \text {, }
$$

and let $a(\not \equiv 0,1, \infty)$ be a small meromorphic function of $f$ and $g$. Then

$$
\bar{N}_{(3}\left(r, \frac{1}{g-a}\right)=S(r, g), \quad \bar{N}_{(3}\left(r, \frac{1}{f-a}\right)=S(r, f) .
$$

Moreover, if $g \notin\left\{\frac{a f}{f+a-1},(1-a) f+a\right.$, af $\}$ or a is a constant then

$$
N_{(3}\left(r, \frac{1}{g-a}\right)=S(r, g)
$$

Theorem C. Under the assumptions of Theorem B, if $N_{2)}\left(r, \frac{1}{g-a}\right) \neq T(r, g)+$ $S(r, g)$, then $\bar{N}\left(r, \frac{1}{g-a}\right)=S(r, g)$, and $f$ and $g$ satisfy one of the three relations in Theorem A.

Remark 1. Yi [14, Lemma 2.6] has proved that if $f$ and $g$ are two distinct nonconstant meromorphic functions sharing $\left(0, k_{1}\right),\left(1, k_{2}\right),\left(\infty, k_{3}\right)$ where $k_{j}(j=$ 
$1,2,3)$ are positive integers satisfying (1.1), then $\bar{N}_{(2}\left(r, \frac{1}{g-a}\right)=S(r, g)$ and $\bar{N}_{(2}\left(r, \frac{1}{f-a}\right)=S(r, f)$, for all $a=0,1, \infty$. That means, $f$ and $g$ share $0,1, \infty$ GCM.

Example 1. Let $f=q \frac{p e^{z}-1}{p e^{2 z}-q}$ and $g=e^{z} \frac{p e^{z}-1}{p e^{2 z}-q}$, where $p$ and $q$ are nonconstant rational functions with $q p \not \equiv 1$. It is readily checked that $f$ and $g$ share $0,1, \infty$ GCM, but they do not share 0,1 or $\infty$ IM (i.e., $f$ and $g$ do not satisfy the condition of Weighted sharing ).

Question 1. If the condition "sharing three values" in Theorems $\mathrm{B}$ and $\mathrm{C}$ is replaced by the condition "sharing three values GCM ", are Theorems B and C still true?

We answer this question by the following results which extend Theorem B and Theorem C.

Theorem 1. Let $f$ and $g$ be two distinct nonconstant meromorphic functions sharing $0,1, \infty G C M$, and let $a(\not \equiv 0,1, \infty)$ be a small meromorphic function of $f$ and $g$. Then the conclusions of Theorem B still hold.

Theorem 2. Let $f$ and $g$ be two distinct nonconstant meromorphic functions sharing $0,1, \infty$ GCM, and let $a(\not \equiv 0,1, \infty)$ be a small meromorphic function of $f$ and $g$. If $\left.N_{2}\right)\left(r, \frac{1}{g-a}\right) \neq T(r, g)+S(r, g)$ then $\bar{N}\left(r, \frac{1}{g-a}\right)=S(r, g)$, and $f$ and $g$ satisfy one of the three relations in Theorem A.

The following corollary applies readily to Theorems 1 and 2 .

Corollary 1. Let $f$ and $g$ be two distinct nonconstant meromorphic functions sharing $0,1, \infty$ GCM. If $a, b(\not \equiv 0,1, \infty)$ are distinct small meromorphic functions of $f$ and $g$, then either $N_{(3}\left(r, \frac{1}{g-a}\right)=S(r, g)$ or $N_{(3}\left(r, \frac{1}{g-b}\right)=S(r, g)$.

Remark 1 tells us that Theorem 1 extends of Theorem B and Theorem 2 extends of Theorem C.

Example 2. Let $f=\left(e^{p}-1\right)^{2}, g=e^{p}-1$ and $a=-1$, where $p$ is a nonconstant polynomial. We see that $f$ and $g$ share 0 GIM. Furthermore, $f$ and $g$ share $1, \infty$ GCM, and $N(r, 1 /(g-a))=0$, but we see that the conclusions of Theorem A fail to hold. This shows that the condition "sharing $0,1, \infty$ GCM" in Theorem 2 is necessary.

\section{Lemmas}

Lemma 1([11]). Let $f$ and $g$ be two nonconstant meromorphic functions sharing $0,1, \infty$ GIM. Then $T(r, f) \leq 3 T(r, g)+S(r, f)$ and $T(r, g) \leq 3 T(r, f)+S(r, g)$. 
The lemma 1 shows that $S(r, f)=S(r, g)$ and we denote them by $S(r)$, unless otherwise stated.

Lemma 2. Let $f$ and $g$ be two distinct nonconstant meromorphic functions sharing $0,1, \infty$ GIM, and let $\alpha=\frac{f-1}{g-1}$ and $H=\frac{f}{g}$. The following statements are equivalent:

(i) $f$ and $g$ share $0,1, \infty G C M$;

(ii) $\bar{N}_{(2}\left(r, \frac{1}{f-a}\right)+\bar{N}_{(2}\left(r, \frac{1}{g-a}\right)=S(r)$, for $a=0,1, \infty$;

(iii) $\bar{N}\left(r, \frac{1}{\alpha-a}\right)+\bar{N}\left(r, \frac{1}{H-a}\right)=S(r)$, for $a=0, \infty$.

Proof. Let

$$
\phi_{1}=\frac{f^{\prime}}{f}-\frac{g^{\prime}}{g}, \quad \phi_{2}=\frac{f^{\prime}}{f-1}-\frac{g^{\prime}}{g-1}, \quad \phi_{3}=\frac{f^{\prime}}{f(f-1)}-\frac{g^{\prime}}{g(g-1)} .
$$

It is clear that if $\phi_{1} \equiv 0$ then $f=A g$, where $A \neq 0,1$ is a constant. Hence, $f$ and $g$ share $0,1, \infty \mathrm{GCM}$, and $\bar{N}\left(r, \frac{1}{f-1}\right)+\bar{N}\left(r, \frac{1}{f-A}\right)=S(r)$. By the second fundamental theorem of Nevanlinna, we get $T(r, f)=\bar{N}\left(r, \frac{1}{f}\right)+S(r)=\bar{N}(r, f)+$ $S(r)$, which gives us $\bar{N}_{(2}\left(r, \frac{1}{f}\right)+\bar{N}_{(2}(r, f)=S(r)$. In fact, one can prove that the lemma is clear when $\phi_{i} \equiv 0(i=2,3)$. Therefore, we consider that $\phi_{i} \not \equiv 0 \quad(i=$ $1,2,3)$.

(i) $\Longrightarrow$ (ii) We first prove that $T\left(r, \phi_{1}\right)=S(r)$. We can easily verify that the poles of $\phi_{1}$ occur at (1) the zeros and poles of $f(2)$ the zeros and poles of $g$. Since the poles of $\phi_{1}$ are simple and $m\left(r, \phi_{1}\right)=S(r)$, then $T\left(r, \phi_{1}\right)=S(r)$. Similarly, $T\left(r, \phi_{i}\right)=S(r)(i=2,3)$.

We may view that if $z$ is a common zero of $f$ and $g$ with the same multiplicity $(\geq 2)$ then $z$ is also a zero of $\phi_{2}$. Consequently, since (i) occurs then

$$
\bar{N}_{(2}\left(r, \frac{1}{f}\right) \leq N\left(r, \frac{1}{\phi_{2}}\right)+S(r) \leq T\left(r, \phi_{2}\right)+S(r)=S(r) .
$$

In the same way, we can prove that

$$
\bar{N}_{(2}\left(r, \frac{1}{f-1}\right)+\bar{N}_{(2}\left(r, \frac{1}{g}\right)+\bar{N}_{(2}\left(r, \frac{1}{g-1}\right)+\bar{N}_{(2}(r, f)+\bar{N}_{(2}(r, g)=S(r) .
$$

(ii) $\Longrightarrow$ (iii) We see $\bar{N}\left(r, \frac{1}{H}\right) \leq \bar{N}_{(2}\left(r, \frac{1}{f}\right)+\bar{N}_{(2}(r, g)+S(r)=S(r)$.

Similarly, $\bar{N}\left(r, \frac{1}{\alpha}\right)+\bar{N}(r, H)+\bar{N}(r, \alpha)=S(r)$.

(iii) $\Longrightarrow$ (i) Since $\phi_{1}=\frac{H^{\prime}}{H}$ and $\phi_{2}=\frac{\alpha^{\prime}}{\alpha}$, it is obvious that $T\left(r, \phi_{i}\right)=S(r), \quad(i=$ 
$1,2,3)$.

Let $z$ be a common zero of $f$ and $g$ with multiplicity $n$ and $m$ respectively. If $n \neq m$, then $z$ is a pole of $\phi_{1}$, but the counting function of those points is equal to $S(r)$, that is, $f$ and $g$ share 0 GCM. Similarly, $f$ and $g$ share $1, \infty$ GCM. This proves Lemma 2.

From the proof of Lemma 2, we deduce the following lemma:

Lemma 3. Let $f$ and $g$ be two distinct nonconstant meromorphic functions sharing $0,1, \infty$ GCM. Suppose that $\phi_{1}=\frac{H^{\prime}}{H}, \phi_{2}=\frac{\alpha^{\prime}}{\alpha}$ and $\phi_{3}=\frac{H_{0}^{\prime}}{H_{0}}$ are not constant functions, where $H_{0}=\frac{\alpha}{H}$. Then $T\left(r, \phi_{i}\right)=S(r), i=1,2,3$.

Lemma 4. Let $f$ and $g$ be nonconstant meromorphic functions sharing $0,1, \infty$ $G C M$ such that $f$ is not a linear transformation of $g$. Then each of the following holds:

(i) $T(r, f)+T(r, g)=N_{0}(r)+\bar{N}\left(r, \frac{1}{g}\right)+\bar{N}(r, g)+\bar{N}\left(r, \frac{1}{g-1}\right)+S(r)$;

(ii) $N_{(2}\left(r, \frac{1}{f-g}\right)=S(r)$;

(iii) $N_{0}\left(r, \frac{1}{g^{\prime}}\right)=\bar{N}_{0}\left(r, \frac{1}{g^{\prime}}\right)+S(r, g), N_{0}\left(r, \frac{1}{f^{\prime}}\right)=\bar{N}_{0}\left(r, \frac{1}{f^{\prime}}\right)+S(r, f), N_{0}(r)=$ $\bar{N}_{0}(r)+S(r)$;

(iv) $T(r, f)=N_{0}(r)+N_{0}\left(r, \frac{1}{g^{\prime}}\right)+S(r), \quad T(r, g)=N_{0}(r)+N_{0}\left(r, \frac{1}{f^{\prime}}\right)+S(r)$;

(v) $N\left(r, \frac{g(g-1)}{f-g}\right)=N(r, g)+N_{0}(r)+S(r)$,

where $N_{0}(r)\left(\bar{N}_{0}(r)\right)$ denotes the counting function of the zeros of $f-g$ which are not the zeros of $g(g-1), 1 / g$ (ignoring multiplicities) and $N_{0}\left(r, \frac{1}{f^{\prime}}\right) \quad\left(\bar{N}_{0}\left(r, \frac{1}{f^{\prime}}\right)\right.$ ) denotes the counting function corresponding to the zeros of $f^{\prime}$ that are not zeros of $f(f-1)$ ( ignoring multiplicities ).

Proof. Since $f$ is not a linear transformation of $g$ then $\alpha, H$ and $H_{0}$ are nonconstant functions, where $\alpha, H$ and $H_{0}$ are defined as in Lemmas 2 and 3. Let $\lambda=\frac{\frac{\alpha^{\prime}}{\alpha}}{\frac{\alpha^{\prime}}{\alpha}-\frac{H^{\prime}}{H}}$. Then from Lemmas 2 and 3 , we see that $\lambda$ is a small function of $f$, and

$$
f=\frac{1-\alpha^{-1}}{H^{-1}-\alpha^{-1}}, \quad g=\frac{1-\alpha}{H-\alpha} .
$$

By (2.2), it is easily verified that

$$
\frac{H_{0}^{\prime}}{H_{0}}(f-\lambda)=\frac{g^{\prime}(g-f)}{g(g-1)} .
$$

Let $F=(f-\lambda)\left(H_{0}-1\right)=\alpha-\lambda H_{0}+\lambda-1$. Then $\frac{F^{\prime}}{F}-\frac{\alpha^{\prime}}{\alpha}=\frac{\frac{\alpha^{\prime}}{\alpha}(\lambda-1)-\lambda^{\prime}}{f-\lambda}$. 
If $\frac{\alpha^{\prime}}{\alpha}(\lambda-1)-\lambda^{\prime} \equiv 0$, then $T(r, \alpha)+T(r, F)=S(r)$. That is, $T\left(r, H_{0}\right)=S(r)$, and by $(2.2)$ we get $T(r, f)=S(r)$, which is impossible. Consequently, we have $\frac{1}{f-\lambda}=$ $\frac{\frac{F^{\prime}}{F}-\frac{\alpha^{\prime}}{\alpha}}{\frac{\alpha^{\prime}}{\alpha}(\lambda-1)-\lambda^{\prime}}$. This formula and Lemmas 2, 3 yield $m\left(r, \frac{1}{f-\lambda}\right)+N_{(2}\left(r, \frac{1}{f-\lambda}\right)=$ $S(r)$, which implies

$$
T(r, f)=N_{1)}\left(r, \frac{1}{f-\lambda}\right)+S(r) .
$$

Let $z$ be a zero of $g^{\prime}$ with multiplicity $n(\geq 2)$ such that it is not the zero of $g(g-1)$. If $z$ is not the pole of $f$, then from (2.3) and (2.4), we deduce that the counting function of those points is equal to $S(r)$.

Consider that $z$ is a pole of $f$ with multiplicity $i(f)(\geq 2)$. Then $z$ is a zero of $\phi_{3}$ with multiplicity $i\left(\phi_{3}\right) \geq \min \{n, i(f)-1\}$. If $n \leq i(f)-1$ then, from Lemma 3 , it is obvious that the counting function of those points is equal to $S(r)$.

Assume that $n>i(f)-1$. If $n=i(f)$ then $2 i\left(\phi_{3}\right) \geq n$; and if $n=i(f)+1$ then $3 i\left(\phi_{3}\right) \geq n$; and if $n>i(f)+1$ then $z$ is a zero of $\frac{H_{0}^{\prime}}{H_{0}}(f-\lambda)$ with multiplicity $\geq n-i(f) \geq 2$. Then from (2.3), (2.4) and Lemma 3, we get that the counting function of those points is equal to $S(r)$. Consequently, we conclude that

$$
N_{0}\left(r, \frac{1}{g^{\prime}}\right)=\bar{N}_{0}\left(r, \frac{1}{g^{\prime}}\right)+S(r, g) .
$$

The proof of the rest (iii) follows from (2.3) and (2.4). Again, the identities (2.3) and (2.4) give us $T(r, f)=N_{1)}\left(r, \frac{1}{f-\lambda}\right)=\bar{N}_{0}\left(r, \frac{1}{g^{\prime}}\right)+\bar{N}_{0}(r)+S(r, g)$, which is (iv). By (iii) and (iv), it is not difficult to show that

$$
N(r, f-g) \leq N(r, f)+N_{(2}(r, g)+S(r) .
$$

By the second fundamental theorem of Nevanlinna, Lemma 2, (2.5) and by using (iv), we note

$$
\begin{aligned}
& T(r, f)+T(r, g) \\
\leq & \bar{N}_{0}\left(r, \frac{1}{g^{\prime}}\right)+\bar{N}_{0}(r)+\bar{N}\left(r, \frac{1}{g}\right)+\bar{N}(r, g)+\bar{N}\left(r, \frac{1}{g-1}\right)-N_{0}\left(r, \frac{1}{g^{\prime}}\right)+S(r) \\
\leq & \bar{N}_{0}(r)+\bar{N}\left(r, \frac{1}{g}\right)+\bar{N}(r, g)+\bar{N}\left(r, \frac{1}{g-1}\right)+S(r) \\
\leq & \bar{N}\left(r, \frac{1}{f-g}\right)+\bar{N}(r, g)+S(r) \leq N\left(r, \frac{1}{f-g}\right)+N_{1)}(r, g)+S(r) \\
\leq & T(r, f-g)+N_{1)}(r, g)+S(r) \\
\leq & m(r, f)+m(r, g)+N(r, f)+N_{(2}(r, g)+N_{1)}(r, g)+S(r) \\
= & T(r, f)+T(r, g)+S(r) .
\end{aligned}
$$


From this we deduce (i) and (ii).

It remains only to prove (v). Let $z_{0}$ be a zero of $\frac{f-g}{g(g-1)}$ with multiplicity $m \geq 1$.

(1) If $z_{0}$ is a zero of $g(g-1)$ then it is a zero of $f-g$ with multiplicity $>m$.

(2) If $z_{0}$ is not the zero of $g(g-1), \frac{1}{g}$ then it is a zero of $f-g$ with multiplicity $m$.

(3) If $z_{0}$ is a pole of $g$ with multiplicity $i(g)$ and it is not a pole of $f$, then $i(g)=m$. Suppose that $z_{0}$ is a pole of $f$ and $g$ with multiplicity $i(f)$ and $i(g)$ respectively.

(4) If $i(g)<i(f)$, then $m=2 i(g)-i(f)$. Thus, $i(g)>1$ and $z_{0}$ must be a zero of $\phi_{3}$ with multiplicity $\geq i(g)-1$, where $\phi_{3} \not \equiv 0$ is defined as in (2.1).

(5) If $i(g)=i(f) \geq 2$ and $z_{0}$ is not the zero of $f-g$ then $m \leq 2 i(g)$ and $z_{0}$ is a zero of $\phi_{3}$ with multiplicity $\geq i(g)-1$.

(6) If $i(g)=i(f) \geq 2$ and $z_{0}$ is a zero of $f-g$ with multiplicity $i(f-g)$ then $m=i(f-g)+2 i(g)$ and $z_{0}$ is a zero of $\phi_{3}$ with multiplicity $\geq i(g)-1$.

We denote by $N_{j}(r)$ the counting function of those zeros of $\frac{f-g}{g(g-1)}$ which fall in the case $(j), j \in\{1,2,3,4,5,6\}$. Therefore, Lemma 2, Lemma 3 , and (ii) and (iii) of Lemma 4, we deduce that $N_{j}(r)=S(r), j \in\{1,4,5,6\}$ and $N_{2}(r)=N_{0}(r)+S(r)$. We denote by $N_{7}(r)$ the counting function of those zeros of $\frac{f-g}{g(g-1)}$ such that every point in that function is a common pole of $f$ and $g$ with multiplicities $i(f)$ and $i(g)$ respectively, and $i(f) \leq i(g)$, each point in that function is counted according to the multiplicities of poles of $g$. Consequently,

$$
N\left(r, \frac{f-g}{g(g-1)}\right)=N_{3}(r)+N_{7}(r)+N_{0}(r)+S(r)=N(r, g)+N_{0}(r)+S(r),
$$

which is (v). This proves Lemma 4.

Lemma $5([7])$. Let $f_{1}$ and $f_{2}$ be nonconstant meromorphic functions satisfying

$\bar{N}\left(r, f_{i}\right)+\bar{N}\left(r, \frac{1}{f_{i}}\right)=S(r), T\left(r, f_{i}\right) \neq S(r), T\left(r, \frac{f_{i}}{f_{j}}\right) \neq S(r), \quad i \neq j, \quad i, j=1,2$.

Let $a_{i}$ and $b_{i}(i=1,2)$ be nonzero small meromorphic functions of $f_{1}$ and $f_{2}$. Then

$T\left(r, a_{1} f_{1}+a_{2} f_{2}\right)=T\left(r, b_{1} f_{1}+b_{2} f_{2}\right)+S(r), m\left(r, a_{1} f_{1}+a_{2} f_{2}\right)=m\left(r, b_{1} f_{1}+b_{2} f_{2}\right)+S(r)$,

where $S(r)=o\left(\max \left\{T\left(r, f_{1}\right), T\left(r, f_{2}\right)\right\}\right)$.

Lemma 6([6]). Let $f_{1}, f_{2}, f_{3}$ be nonconstant meromorphic functions such that $f_{1}+f_{2}+f_{3} \equiv 1$. If $f_{1}, f_{2}, f_{3}$ are linearly independent, then

$T\left(r, f_{1}\right) \leq N_{2}\left(r, \frac{1}{f_{1}}\right)+N_{2}\left(r, \frac{1}{f_{2}}\right)+N_{2}\left(r, \frac{1}{f_{3}}\right)+\bar{N}\left(r, f_{1}\right)+\bar{N}\left(r, f_{2}\right)+\bar{N}\left(r, f_{3}\right)+S(r)$,

where $N_{2}\left(r, f_{i}\right)=\bar{N}\left(r, f_{i}\right)+\bar{N}_{(2}\left(r, f_{i}\right)$ and $S(r)=o\left(\max \left\{T\left(r, f_{1}\right), T\left(r, f_{2}\right), T\left(r, f_{3}\right)\right\}\right)$. 
Lemma $7([\mathbf{1 6}])$. Let $f_{1}$ and $f_{2}$ be two distinct nonconstant meromorphic functions satisfying $\bar{N}\left(r, f_{i}\right)+\bar{N}\left(r, \frac{1}{f_{i}}\right)=S(r), i=1,2$. Then either $N_{0}\left(r, 1, f_{1}, f_{2}\right)=$ $S\left(r, f_{1}, f_{2}\right)$ or there exist two integers $s, t(|s|+|t|>0)$ such that $f_{1}^{s} f_{2}^{t} \equiv$ 1. Here $N_{0}\left(r, 1, f_{1}, f_{2}\right)$ is the counting function of the common 1-points of $f_{1}$ and $f_{2}$, each point in that function is counted only once, and $S\left(r, f_{1}, f_{2}\right)=$ $\max \left\{S\left(r, f_{1}\right), S\left(r, f_{2}\right)\right\}$.

The proof of the following lemma is omitted, since it can be proved by the similar lines of Lemma 7 in [16].

Lemma 8. Let $f$ and $g$ be nonconstant meromorphic functions sharing $0,1, \infty$ $G C M$. If $f$ is a linear transformation of $g$, then $f$ and $g$ assume one of the following relations:

(i) $g \equiv f$; (ii) $g+f \equiv 1$; (iii) $(g-1)(f-1) \equiv 1$; (iv) $g f \equiv 1$; (v) $(g-A)(f+A-1) \equiv$ $A(1-A)$; (vi) $g+(A-1) f \equiv A$; (vii) $g \equiv A f$, where $A \notin\{0,1\}$ is a constant.

\section{Proofs of theorems 1, 2 and corollary 1}

3.1. Proofs of theorems 1, 2. We only prove (1.2) for $g$, because (1.2) for $f$ can be proved in a similar manner. If $f$ is a linear transformation of $g$, from Lemma 8 we see that there are $a_{1}, a_{2} \in \mathbb{C} \bigcup\{\infty\}$ such that $a_{1} \neq a_{2}$ and $\bar{N}\left(r, \frac{1}{g-a_{1}}\right)+\bar{N}\left(r, \frac{1}{g-a_{2}}\right)=S(r)$. Hence, if $a \notin\left\{a_{1}, a_{2}\right\}$ then, by Nevanlinna's three small functions theorem, we have $T(r, g)=\bar{N}_{1)}\left(r, \frac{1}{g-a}\right)+S(r)$, which implies (1.3), otherwise, the possibilities (i)-(iv) of Lemma 8 do not occur, and hence, the conclusions of Theorems 1 and 2 follow from the possibilities (v)-(vii) of Lemma 8. Therefore, we assume that $f$ is not a linear transformation of $g$. It is evident from Lemma 1 and (2.2) that

$$
S(r)=\max \{S(r, \alpha), S(r, H)\} .
$$

Assume that $T(r, \alpha)=S(r)$. Then from (2.2), we have $g-a=-a y \frac{H-\alpha-\frac{1-\alpha}{a}}{H-\alpha}$. If $\alpha+\frac{1-\alpha}{a} \not \equiv 0$ then from this, (iii) of Lemma 2, (2.2), (3.1) and by applying Nevanlinna's three small functions, we get

$$
T(r, g)=T(r, H)+S(r)=\bar{N}\left(r, \frac{1}{H-\alpha-\frac{1-a}{a}}\right)+S(r)=\bar{N}\left(r, \frac{1}{g-a}\right)+S(r),
$$

which implies (1.3). We note that the case $\alpha+\frac{1-\alpha}{a} \equiv 0$ gives (ii) of Theorem A, and the remaining conclusions of Theorem 1 and 2 follow from Lemma 2 .

Similarly, if $T(r, H)=S(r)$ or $T\left(r, \frac{\alpha}{H}\right)=S(r)$, then we deduce the conclusions 
of Theorems 1 and 2. We may assume that $T(r, H), T(r, \alpha)$ and $T\left(r, \frac{\alpha}{H}\right)$ are not equal to $S(r)$. Let us put $f_{1}=-G, f_{2}=(1-a) \alpha, f_{3}=a H$, from $(2.2)$ we have

$$
G=(g-a)(\alpha-H)=(1-a) \alpha+a H-1
$$

and

$$
f_{1}+f_{2}+f_{3}=1 \text {. }
$$

Suppose that $T\left(r, f_{1}\right)=S(r)$. Then from (3.2), we get $H=\frac{-f_{1}+1-(1-a) \alpha}{a}$. If $f_{1} \not \equiv 1$ then from Lemma 2 and by using the second fundamental theorem of Nevanlinna, we observe that

$$
T(r, \alpha)=\bar{N}\left(r, \frac{1}{-f_{1}+1-(1-a) \alpha}\right)+S(r) \leq \bar{N}\left(r, \frac{1}{H}\right)+S(r)=S(r),
$$

which is a contradiction. Thus $f_{1} \equiv 1$, which implies (i) of Theorem A, and the remaining conclusions of Theorems 1 and 2 follow from Lemma 2. Therefore, it is enough to prove Theorems 1 and 2 , when $T\left(r, f_{i}\right)(i=1,2,3)$ are not equal to $S(r)$. First, we claim

$$
T\left(r, f_{1}\right)=N_{2)}\left(r, \frac{1}{f_{1}}\right)+S(r) .
$$

In order to prove (3.4), we suppose that $f_{1}, f_{2}$ and $f_{3}$ are linearly independent. Evidently, from (iii) of Lemma 2, (3.3) and by applying Lemma 6 we obtain that

$$
T\left(r, f_{1}\right) \leq N_{2}\left(r, \frac{1}{f_{1}}\right)+S(r) \leq N\left(r, \frac{1}{f_{1}}\right)+S(r),
$$

which is (3.4).

Suppose that $f_{1}, f_{2}$ and $f_{3}$ are linearly dependent. Then there exist constants $c_{1}, c_{2}$ and $c_{3}$ (not all are zeros) such that

$$
c_{1} f_{1}+c_{2} f_{2}+c_{3} f_{3} \equiv 0 .
$$

Let us prove that $c_{1}=0$. Otherwise, eliminating $f_{1}$ from (3.3) and (3.5), we get $\left(1-\frac{c_{2}}{c_{1}}\right) f_{2}+\left(1-\frac{c_{3}}{c_{1}}\right) f_{3} \equiv 1$. From this, (iii) of Lemma 2 and by applying the second fundamental theorem of Nevanlinna, we get $T\left(r, f_{2}\right)=S(r)$, which is a contradiction.

Therefore, $c_{1}=0$ and $c_{2} c_{3} \neq 0$. Identities (3.3) and (3.5) imply that $c_{2} f_{1}+\left(c_{2}-\right.$ $\left.c_{3}\right) f_{3}=c_{2}$, and from this and (iii) of Lemma 2 , we obtain that $\bar{N}\left(r, \frac{1}{f_{1}-1}\right)=S(r)$. Again, (iii) of Lemma 2 and (3.2) yield that $\bar{N}\left(r, f_{1}\right)=S(r)$. Therefore, by using Nevanlinna's second fundamental theorem, we get (3.4) and this completes the proof 
of (3.4).

The formula (3.2) can be rewritten as

$$
g-a=\frac{(1-a) \alpha+a H-1}{\alpha-H}=\frac{G}{\alpha-H} .
$$

It follows from Lemma 5 and (3.2) that

$$
T(r, G)=T(r,(1-a) \alpha+a H)+S(r)=T(r, \alpha-H)+S(r) .
$$

Again, by using Lemma 5 and (3.2), we obtain

$$
N(r, G)=N(r,(1-a) \alpha+a H)+S(r)=N(r, \alpha-H)+S(r) .
$$

But we know $\alpha-H=\frac{f-g}{g(g-1)}$. Then this, (v) of Lemma 4, (3.6) and (3.8) yield

$$
\begin{aligned}
N\left(r, \frac{1}{g-a}\right) & =N\left(r, \frac{1}{G}\right)-N\left(r, \frac{1}{\alpha-H}\right)+N(r, g)+S(r) \\
& =N\left(r, \frac{1}{G}\right)-N_{0}(r)+S(r) .
\end{aligned}
$$

Since $g-a=-\frac{1}{\alpha-H}+1-a+\frac{1}{\frac{\alpha}{H}-1}$ and $m\left(r, \frac{1}{\frac{\alpha}{H}-1}\right)=S(r)$, then $m\left(r, \frac{1}{\alpha-H}\right)=$ $m(r, g)+S(r)$. From this, (3.4), (3.8) and (3.9), we get

$$
\begin{aligned}
N\left(r, \frac{1}{g-a}\right) & =m\left(r, \frac{1}{\alpha-H}\right)+N(r, g)+S(r) \\
& =m(r, g)+N(r, g)+S(r)=T(r, g)+S(r) .
\end{aligned}
$$

By (3.4) and (3.6), it is not difficult to check

$$
N_{(3}\left(r, \frac{1}{g-a}\right)=N_{(3}^{*}\left(r, \frac{1}{g-a}\right)+S(r),
$$

where $N_{(3}^{*}\left(r, \frac{1}{g-a}\right)$ is the counting function of the zeros of $g-a$ with multiplicity $\geq 3$ which are the poles of $\alpha-H$, the zeros of $g-a$ are counted according to their multiplicities.

It remains to prove (1.3). To prove this, we discuss the following two cases:

Case 1. Suppose $N_{0}(r) \neq S(r)$, where $N_{0}(r)$ is defined as in Lemma 4. It follows from (3.1) and (iii) of Lemma 4 that

$$
N_{0}(r)=N_{0}(r, 1, \alpha, H)+S(r) .
$$

From (3.12), one can apply Lemma 7 to $\alpha$ and $H$ that there exist two integers $s, t(|s|+|t|>0)$ such that $\alpha^{t} H^{s} \equiv 1$. Therefore,

$$
f^{s}(f-1)^{t}=g^{s}(g-1)^{t} .
$$


Let $z_{0}$ be a zero of $g-a$ with multiplicity $i(g-a) \geq 3$ such that it is a pole of $\alpha-H$ with multiplicity $i(\alpha-H)$.

Subcase 1.1. Assume that $z_{0}$ is a pole of $g$ with multiplicity $i(g)$. Since $s+t \neq 0$, if $z_{0}$ is a pole of $f$ with multiplicity $i(f)$ then, by using (3.13), we get $i(f)=i(g)$, and hence, $z_{0}$ is not the pole of $\alpha-H$. It is readily checked that if $z_{0}$ is a zero of $f(f-1)$, then $z_{0}$ is not the pole of $\alpha-H$, which is a contradiction. Consequently, $z_{0}$ is neither the pole of $f$ nor the zero of $f(f-1)$, from (3.13) it follows that this possibility does not occur.

Subcase 1.2. Assume that $z_{0}$ is a zero of $g$ (or $g-1$ ) with multiplicity $i(g)$ ( or $i(g-1)$ ). Then $z_{0}$ must be a zero of $a$ (or $\left.a-1\right)$ with multiplicity $i(a)$ (or $i(a-1)$ ) . If $i(g) \neq i(a)($ or $i(g-1) \neq i(a-1))$, then $i(g-a) \leq i(a)$ (or $i(g-a) \leq i(a-1))$. Suppose that $i(g)=i(a)$ (or $i(g-1)=i(a-1))$. If $z_{0}$ is a zero of $G$ with multiplicity $i(G)$ then, from (3.6), we get $i(g-a) \leq i(G)+i(\alpha-H)$. If $z_{0}$ is not the zero of $G$ then $i(g-a) \leq i(\alpha-H)$.

If $g\left(z_{0}\right) \neq 0,1, \infty$ then, from (3.13), we get $f\left(z_{0}\right) \neq 0,1, \infty$, that is, $z_{0}$ is not the pole of $\alpha-H$, which is a contradiction. Consequently, from (3.11), the subcases 1.1 and 1.2 , and by using (3.4), we conclude

$$
N_{(3}\left(r, \frac{1}{g-a}\right) \leq N_{0}^{*}(r, \alpha-H)+N_{1}^{*}(r, \alpha-H)+S(r),
$$

where $N_{0}^{*}(r, \alpha-H)$ (or $\left.N_{1}^{*}(r, \alpha-H)\right)$ is the counting function of the poles of $\alpha-H$ that are the common zeros of $g$ and $a$ (or $g-1$ and $a-1$ ) with the same multiplicities, the poles of $\alpha-H$ are counted according to their multiplicities.

Let $z_{0}$ be a pole of $\alpha-H$ with multiplicity $i(\alpha-H)$ such that $z_{0}$ is a common zero of $g$ and $a$ with multiplicity $i(g)$ and $i(a)$ respectively, and $i(a)=i(g)$. From (3.13), if $z_{0}$ is a zero of $f$ with multiplicity $i(f)$ then $i(f)=i(g)$, and hence, $z_{0}$ is not the pole of $\alpha-H$. Therefore, from (3.13) that either $z_{0}$ is a zero of $f-1$ or else $z_{0}$ is a pole of $f$ with multiplicity $i(f)$. If the first possibility occurs then $i(\alpha-H)=i(a)$. Otherwise, we suppose that the second possibility occurs. Then, from (3.13), we deduce $-(s+t) i(f)=s i(g)=s i(a)$ and $i(\alpha-H) \leq i(f)+i(g)$ which imply $i(\alpha-H) \leq(t /(s+t)) i(a)$. From this illustration, we deduce that $N_{0}^{*}(r, \alpha-H)=S(r)$. Similarly, $N_{1}^{*}(r, \alpha-H)=S(r)$. Therefore, (3.14) gives (1.3). Case 2. Suppose $N_{0}(r)=S(r)$. Let $z_{0}$ be a zero of $G$ with multiplicity $i(G) \leq 2$ such that $a\left(z_{0}\right) \neq 0,1, \infty$. Assume that $z_{0}$ is a zero of $\alpha-H=\frac{f-g}{g(g-1)}$.

If $z_{0}$ is a simple zero of $g(g-1)$ then it is a zero of $f-g$ with multiplicity $\geq 2$. Since $z_{0}$ is a zero of $G$, therefore, if $z_{0}$ is a simple pole of $g$ and $f$ then $z_{0}$ must be a zero of $\alpha-H$ with multiplicity $\geq 2$. Since $\bar{N}_{(2}(r, 1 /(\alpha-H))=S(r)$, we deduce that the counting function of these points is equal to $S(r)$.

If $z_{0}$ is not any zero of $g(g-1), 1 / g$ then $z_{0}$ must be a zero of $f-g$.

Suppose that $z_{0}$ is a pole of $\alpha-H$. Since $z_{0}$ is a zero of $G$, then we get that if $z_{0}$ is a simple zero of $g(g-1)$, then (3.6) leads us that $z_{0}$ must be a zero of $g-a$, which is a contradiction, because $a\left(z_{0}\right) \neq 0,1, \infty$. Hence, we deduce that the counting function of these points is equal to $S(r)$. 
If $z_{0}$ is not the zero of $\alpha-H$ or $\frac{1}{\alpha-H}$, then $z_{0}$ is a zero of $g-a$ with multiplicity $i(G)$.

It follows from the above, Lemmas 2, 3, (ii) and (iii) of Lemma 4 and (3.4) that $N_{2)}\left(r, \frac{1}{g-a}\right)=N_{2)}\left(r, \frac{1}{G}\right)+S(r)$. By (3.4) and (3.9), we obtain that $N_{(3}\left(r, \frac{1}{g-a}\right)=$ $S(r)$, which is (1.3). By (3.10), we see that the condition $N_{2)}\left(r, \frac{1}{g-a}\right) \neq T(r, g)+$ $S(r)$ in Theorem 2 does not occur.

Suppose that $g \in\left\{\frac{a f}{f+a-1},(1-a) f+a, a f\right\}$ and $a$ is a constant. Firstly, let $g=a f$. If $z$ is a zero of $g-a$ with multiplicity $\geq 3$ then $z$ is a zero of $g^{\prime}$ with multiplicity $\geq 2$. Consequently, we deduce (1.3) from (iii) of Lemma 4 . If $g=(1-a) f+a\left(\right.$ or $\left.g=\frac{a f}{f+a-1}\right)$, we put $G=1-g, F=1-f, b=1-a$ (or $G=1-(1 / g), F=1-(1 / f), b=1-(1 / a))$ to obtain $G=b F$, and $F$ and $G$ share $0,1, \infty$ GMC. From the first case, we get (1.3). The proofs of Theorems 1 and 2 have completed.

\subsection{Proof of corollary 1. If}

$$
g \in\left\{\frac{a f}{f+a-1},(1-a) f+a, a f\right\} \text { and } g \in\left\{\frac{b f}{f+b-1},(1-b) f+b, b f\right\},
$$

then we obtain a contradiction. Otherwise, Corollary 1 follows from Theorem 1. The proof of Corollary 1 has completed.

\section{Applications of the main results}

Nevanlinna four values theorem (see [11], Theorem 4.1) says that if two distinct nonconstant meromorphic functions $f$ and $g$ share four values $\mathrm{CM}$, then $f$ is a fractional linear transformation of $g$. The condition "share four values CM" has been weakened to " $f$ and $g$ share two values CM and two values IM" by Gundersen's theorem (see [3]).

Definition 3. Let $a \in \mathbb{C} \bigcup\{\infty\}$. If $f(z)=a$ when $g(z)=a$, then we denote this property by $g(z)=b \Rightarrow f(z)=a$.

We note that the definition $g(z)=b \Rightarrow f(z)=a$ implies to $g(z)=b \Rightarrow f(z)=a$.

Definition 4. Let $k$ be a positive integer, and let $a$ be a small function of $f$. We denote by $\bar{E}(a, f)$ the set of distinct zeros of $f(z)-a$ (ignoring multiplicities), and by $\bar{E}_{k)}(a, f)$ the set of distinct zeros of $f(z)-a$ with multiplicity $\leq k$ (ignoring multiplicities).

In 1989, Brosch [2] proved the following theorem which is an extension of a 
result of $\mathrm{H}$. Ueda [9].

Theorem D. Let $f$ and $g$ be two nonconstant meromorphic functions sharing $0,1, \infty C M$ and let $a \notin\{0,1\}$ be a finite complex number. If $f=a \Rightarrow g=a$, then $f$ is a fractional linear transformation of $g$.

As an application of Theorem 1 and Theorem 2, we extend Theorem D by showing the following result:

Theorem 3. Let $f$ and $g$ be nonconstant meromorphic functions sharing $0,1, \infty$ $G C M$, and let $a(\equiv 0,1, \infty)$ be a small meromorphic function of $f$ and $g$ such that $g=a \Rightarrow f=a$ or $\bar{E}_{2)}(a, g) \subseteq \bar{E}(a, f)$. Then one assumes of the following relations: (i) $g \equiv f$; (ii) $g+f \equiv 1$ with $a=1 / 2$; (iii) $(g-1)(f-1) \equiv 1$ with $a=2$; (iv) $g f \equiv 1$ with $a=-1$; (v) $(g-a)(f+a-1) \equiv a(1-a)$; (vi) $g+(a-1) f \equiv a$; (vii) $g \equiv a f$.

From Theorem 3, one can be checked the following corollary:

Corollary 2. Let $f$ and $g$ be two nonconstant meromorphic functions sharing $0,1, \infty G C M$, and let $a(\equiv \equiv, 1, \infty,-1,2,1 / 2)$ be a small meromorphic function of $f$ and $g$. If $f$ and $g$ share $a$ GIM or $\bar{E}_{2)}(a, g)=\bar{E}_{2)}(a, f)$, then $f \equiv g$.

To prove Theorem 3, we need the following fact which extends Theorems 1 and 2 in [16].

Lemma 9. Let $f$ and $g$ be two distinct nonconstant meromorphic functions sharing $0,1, \infty G C M$ such that $N_{0}(r) \neq S(r)$.

(i) $f$ is a linear transformation of $g$ if and only if $T(r, f)=N_{0}(r)+S(r)$.

(ii) $f$ is not any linear transformation of $g$ if and only if $N_{0}(r) \leq \frac{1}{2} T(r, f)+S(r)$. Furthermore, if (ii) occurs then there is a nonconstant meromorphic $h$ such that

(4.1) $\bar{N}\left(r, \frac{1}{h}\right)+\bar{N}(r, h)=S(r), \quad N_{0}(r)=T(r, h)+S(r), \quad N_{0}(r)=\frac{1}{k} T(r, f)+S(r)$,

and $f$ and $g$ satisfy one of the following relations:

(a) $g=\frac{h^{r}-1}{h^{k+1}-1}, \quad f=\frac{h^{-r}-1}{h^{-(k+1)}-1}$;

(b) $g=\frac{h^{k+1}-1}{h^{k+1-r}-1}, \quad f=\frac{h^{-(k+1)}-1}{h^{-(k+1-r)}-1}$;

(c) $g=\frac{h^{r}-1}{h^{-(k+1-r)}-1}, \quad f=\frac{h^{-r}-1}{h^{(k+1-r)}-1}$,

where $r$ and $k(\geq 2)$ are positive integers such that $r$ and $k+1$ are relatively prime and $1 \leq r \leq k$.

Proof. According to the assumptions of Lemma 9, then Lemma 8 leads us that if $f$ is a linear transformation of $g$ then $T(r, f)=N_{0}(r)+S(r)$. 
Suppose that $f$ is not any linear transformation of $g$. Since $N_{0}(r) \neq S(r)$. From (3.12) and by applying Lemma 7 we deduce that there exist two integers $s, t(|s|+$ $|t|>0)$ such that $\alpha^{t} H^{s} \equiv 1$. Hence, from (3.13), we get $T(r, f)=T(r, g)+S(r)$. Without loss of generality, we can assume that $s$ and $t$ are relatively prime and $s>0$, because $N_{0}(r) \neq S(r)$. Hence, there exist two integers $u$ and $v$ such that $u s+v t=1$. If we let $h=\alpha^{-u} H^{v}$ then from (2.2) and lemma 2 , we have the first relation in (4.1) and

$$
g=\frac{h^{s}-1}{h^{s+t}-1}, \quad f=\frac{h^{-s}-1}{h^{-(s+t)}-1} .
$$

Since $s$ and $t$ are relatively prime, then $\frac{h^{s}-1}{h-1}, \frac{h^{s+t}-1}{h-1}$ have no common zeros. If $z$ is a zero of $f-g$ such that it is not the zero of $f(f-1), 1 / f$ then $z$ is a common zero of $H-1$ and $\alpha-1$ that is, $z$ is also a zero of $h-1$. It follows that

$$
N_{0}(r) \leq \bar{N}\left(r, \frac{1}{h-1}\right)+S(r)=T(r, h)+S(r) .
$$

Let $z$ is a zero of $h-1$ such that it is not a zero of $f(f-1), 1 / f$ then $z$ is a common zero of $H-1$ and $\alpha-1$ that is $T(r, h)+S(r)=\bar{N}\left(r, \frac{1}{h-1}\right) \leq N_{0}(r)+S(r)$. The last two inequalities imply the second relation in (4.1).

Then three cases are needed to be discussed.

Case 1. Suppose that $t$ is a positive. If $s+t=2$, then $s=t=1$, and from (3.13) we get that $f$ is a linear transformation of $g$ which is a contradiction. So that $s+t>2$. From 4.2, we note that $T(r, g)=(s+t-1) T(r, h)+S(r)$, which implies

$$
N_{0}(r)=\frac{1}{s+t-1} T(r, g)+S(r) \leq \frac{1}{2} T(r, g)+S(r) .
$$

In this case, we take $k=s+t-1$ and $r=s$. Then the case (a) in the lemma 9 follows from (4.2).

Case 2. Suppose that $t<0$ and $s+t>0$. If $s=2$, then $t=-1$, and from (3.13) we get that $f$ is a linear transformation of $g$ which is a contradiction. We assume that $s>2$. It follows from 4.2 that $T(r, g)=(s-1) T(r, h)+S(r)$, that is,

$$
N_{0}(r)=\frac{1}{s-1} T(r, g)+S(r) \leq \frac{1}{2} T(r, g)+S(r) .
$$

Here, we take $k=s-1$ and $r=-t$ to obtain the case (b) in the lemma 9 , by using (4.2).

Case 3. Suppose that $t<0$ and $s+t<0$. Obviously, $-t \geq 2$. If $-t=2$, then $s=1$, and from (3.13) we get that $f$ is a linear transformation of $g$. Suppose that $-t>2$. Then (4.2) gives us that $T(r, g)=(-t-1) T(r, h)+S(r)$, which implies

$$
N_{0}(r)=-\frac{1}{t+1} T(r, g)+S(r) \leq \frac{1}{2} T(r, g)+S(r) .
$$


If we put $k=-(t+1)$ and $r=s$, then we have case (c) in the lemma 9. It is easy to prove that $r$ and $k$ are done in the cases a, b, c. If $T(r, f)=N_{0}(r)+S(r)$ and $f$ is not any linear transformation of $g$, then

$$
N_{0}(r) \leq \frac{1}{2} T(r, f)+S(r),
$$

which is a contradiction. That is, if $T(r, f)=N_{0}(r)+S(r)$, then $f$ is a linear transformation of $g$, which completes the proof (i). Now, if $N_{0}(r) \leq \frac{1}{2} T(r, f)+S(r)$ then, from (i), we deduce that $f$ is not any linear transformation of $g$ and this completes the proof (ii). This proves Lemma 9.

Proof of Theorem 3. It is not difficult to check that if $f$ is a fractional linear transformation of $g$, then Theorem 3 immediately follows from Lemma 8. Therefore, we prove Theorem 3 when $f$ is not a fractional linear transformation of $g$. By utilizing Theorem 1 , it is obviously that if $g=a \Rightarrow f=a$ or $\bar{E}_{2)}(a, g) \subseteq \bar{E}(a, f)$ then

$$
\bar{N}\left(r, \frac{1}{g-a}\right) \leq N_{0}(r)+S(r) .
$$

Suppose that $g \notin\left\{\frac{a f}{f+a-1},(1-a) f+a, a f\right\}$. Then from Theorems 1 and 2 , we get

$$
T(r, g)=N_{2)}\left(r, \frac{1}{g-a}\right)+S(r) .
$$

Similarly to (2.2) and (2.3), we get

$$
\begin{gathered}
-\frac{H_{0}^{\prime}}{H_{0}}(g-\lambda)=\frac{f^{\prime}(f-g)}{f(f-1)}, \\
T(r, g)=N_{1)}\left(r, \frac{1}{g-\lambda}\right)+S(r),
\end{gathered}
$$

where $\lambda=\frac{\frac{\alpha^{\prime}}{\alpha}}{\frac{\alpha^{\prime}}{\alpha}-\frac{H^{\prime}}{H}}$. From (4.3), (4.6) and Lemma 9, we deduce $\lambda \not \equiv a$.

Let $z_{0}$ be a common zero of $g-a$ and $f-a$ such that $a\left(z_{0}\right) \neq 0,1, \infty, \lambda\left(z_{0}\right) \neq 0, \infty$ and $\frac{H_{0}^{\prime}}{H_{0}}\left(z_{0}\right) \neq 0, \infty$. Hence, the right-hand side of (4.5) must be a zero at $z_{0}$, which yields that $g-\lambda$ has a zero at $z_{0}$, so that $z_{0}$ must be a zero of $\lambda-a$. Consequently, from the condition $g=a \Rightarrow f=a$ or $\bar{E}_{2)}(a, g) \subseteq \bar{E}(a, f)$, we get $\bar{N}(r, 1 /(g-a))=S(r)$, and from (4.4) it follows $T(r, g)=S(r)$, which is a contradiction. Therefore, $g \in\left\{\frac{a f}{f+a-1},(1-a) f+a, a f\right\}$. This proves Theorem 3 .

Acknowledgement. The author thanks the anonymous for his/her helpful suggestions. 


\section{References}

[1] T. C. Alzahary, Small functions of meromorphic functions sharing three values with finite weights, Indian J. Pure Appl. Math., 38(2007), 305-316.

[2] G. Brosch, Eindeutigkeitssätze für Meromorphe Funktionen, Thesis Techincal of Aachen, 1989.

[3] G. Gundersen, Meromorphic functions that share four values, Trans. Amer. Math. Soc., 277(1983), 545-567.

[4] W. K. Hayman, Meromorphic Functions, The Clarendon Press, Oxford, 1964.

[5] I. Lahiri, On a result of Ozawa concerning uniqueness of meromorphic functions II, J. Math. Anal. Appl., 283(2003), 66-76.

[6] P. Li and C. C. Yang, Some further results on the unique range sets of meromorphic functions, Kodai Math. J., 18(1995), 437-450.

[7] P. Li and C. C. Yang, On the characteristic of meromorphic functions that share three values CM, J. Math. Anal. Appl., 220(1998), 132-145.

[8] E. Mues, Meromorphic Functions sharing four values, Complex Variables, 12(1989), 169-179.

[9] H. Ueda, Unicity theorems for meromorphic or entire functions, Kodai Math. J., 3(1980), 457-471.

[10] H. Ueda, Unicity thorems for meromorphic or entire functions II, Kodai Mathematical Journal, 6(1983), 26-36.

[11] C. C. Yang and H. X. Yi, Uniqueness Theory of Meromorphic Functions, Kluwer Academic Publishers Dordrecht-Bosten-London, 2003.

[12] S. Z. Ye, Uniqueness of meromorphic functions that share three values, Kodai Math. J., 15(1992), 236-243.

[13] H. X. Yi, Unicity theorems for meromorphic functions that share three values, Kodai Math. J., 18(1995), 300-314.

[14] H. X. Yi, Meromorphic functions with weighted sharing of three values, Complex Variables, 50(2005), 923-934.

[15] W. J. Yuan and H. G. Tain, Unicity results for meromorphic functions sharing small functions, Indian J. Pure Appl. Math., 32(2001), 1411-1419.

[16] Q. C. Zhang, Meromorphic functions sharing three values, Indian J. Pure Appl. Math., 30(1999), 667-682. 